\title{
Excitonic Condensation under Spin-Orbit Coupling and BEC-BCS Crossover
}

\author{
T. Hakioğlu ${ }^{1}$ and Mehmet Şahin ${ }^{2}$ \\ ${ }^{1}$ Department of Physics and National Nanotechnology Research Center, Bilkent University, 06800 Ankara, Turkey \\ ${ }^{2}$ Department of Physics, Faculty of Sciences and Arts, Selçuk University, Kampus 42075 Konya, Turkey
}

(Received 16 May 2006; published 20 April 2007)

\begin{abstract}
The condensation of electron-hole pairs is studied at zero temperature and in the presence of a weak spin-orbit coupling (SOC) in coupled quantum wells. Under realistic conditions, a perturbative SOC can have observable effects in the order parameter of the condensate. First, the fermion exchange symmetry is absent. As a result, the condensate spin has no definite parity. Additionally, the excitonic SOC breaks the rotational symmetry yielding a complex order parameter in an unconventional way; i.e., the phase pattern of the order parameter is a function of the condensate density. This is manifested through finite offdiagonal components of the static spin susceptibility, suggesting a new experimental method to confirm an excitonic condensate.
\end{abstract}

DOI: 10.1103/PhysRevLett.98.166405

A rich variety of low temperature collective phases had been proposed for semiconductors in the 1960s. Condensation of electron-hole $(e-h)$ pairs was studied primarily by Moskalenko, Blatt et al., and the group led by Keldysh [1]. As the excitonic density is increased, these phases range from the BEC to a BCS type ground state and eventually to the $e-h$ liquid [2-4]. Initially, the experimental progress was slow given the difficulties in producing sufficiently long-lived exciton pairs. One of the earliest experiments was carried out by Snoke et al. [5] and Hara et al. [6] on $\mathrm{Cu}_{2} \mathrm{O}$ on 3D samples. The difficulties were overcome by using indirect excitonic transitions [7]. Still longer lifetimes were obtained by containing the two-dimensional $(e)$ and $(h)$ gases (2DEG and 2DHG) separately in Coulomb coupled QWs with a stabilizing $E$ field [8]. Currently, coupled QWs with lifetimes in the microsecond range provide optimum conditions for observing this long proposed state [9].

Here we investigate exciton condensation (EC) in coupled quantum wells $(\mathrm{QW})$ in the presence of a weak in-plane Rashba spin-orbit coupling (SOC) [10]. Here, in contrast to the conventional pairing between identical fermions [11], the only manifested symmetry is time reversal. The $e-h$ exchange symmetry is absent and the parity of the condensate mixes with the condensate spin, disabling the conventional classification schemes [12]. In turn, there is no relation between the parity and the spin of the condensate. Another crucial difference from identical fermion pairing is that the hole SOC breaks the underlying symmetry of the electron SOC-known as $C_{\infty v}$ - and the corresponding complex excitonic order parameter in the up-down spin channel develops an unconventional phase pattern. This can be measured in the off-diagonal components of the static spin susceptibility which may be crucial as a complementary method for identifying the excitonic condensate.

The model geometry studied here is closely related to that of Zhu et al. $[13,14]$ as illustrated in Fig. 1. The $(e)$ and the $(h)$ QWs are separated by a high tunneling barrier of
PACS numbers: 71.35.Gg, 03.75.Lm, 71.35.Lk, 71.70.Ej

thickness $d$ ( $d \simeq 100 \AA$ A here). Although typical external $E$ fields (for instance, Ref. [8]) are in the range of $3-5 \mathrm{kV} / \mathrm{cm}$, the intrinsic fields due to doping can be as high as $100-200 \mathrm{kV} / \mathrm{cm}$, e.g., Ref. [15]. In this case, it is known that the Rashba SOC is the dominant mechanism for the splitting of the energy bands [16]. High tunability factors of the SOC by $E$ fields was previously demonstrated [17-20] and the efforts toward much higher tunabilities are crucial for potential device applications [21].

The mechanism of EC is the interband attractive Coulomb interaction. We consider equal electron and hole densities and the tunneling is negligible [9]. The intraband Coulomb strengths for a typical concentration $n_{x} \simeq$ $10^{11} \mathrm{~cm}^{-2}$ are $V^{e e}=V^{h h}=2 \pi e^{2} /\left(\epsilon r_{e e}\right) \simeq 4-5 \mathrm{meV}$. The layer separation $d \simeq 1$ in units of the effective Bohr radius $a_{e}^{*}=\hbar^{2} \kappa /\left(e^{2} m_{e}^{*}\right) \simeq 100 \AA$. The strength of the Coulomb interaction between the layers is $V^{e h}=2 \pi e^{2} /\left(\epsilon r_{e h}\right) \simeq$ 1-2 meV. The $r_{e e}$ and $r_{e h}$ are the average $e-e$ (or $\left.h-h\right)$

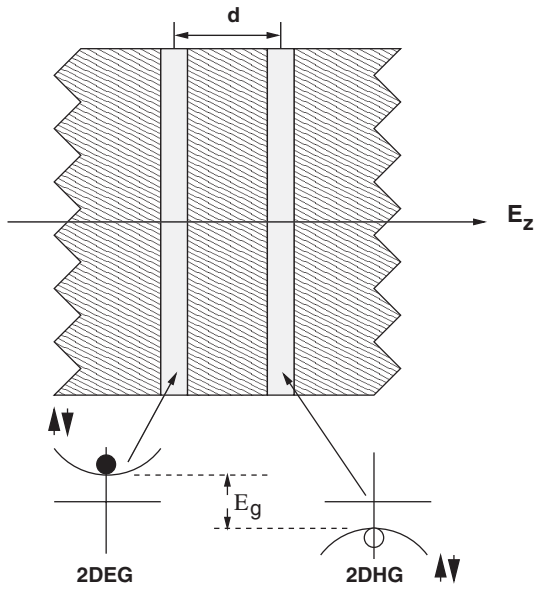

FIG. 1. The double-well geometry in $x-y$ plane. The 2DEG and the $2 \mathrm{DHG}$ are produced within the GaAs wells inserted in high AlGaAs tunneling barriers. We ignore the well widths in this work. The spin-degenerate conduction and valence subbands are considered within the parabolic approximation. 
and $e$ - $h$ separations. Here the SOC is weak at typical densities and treated perturbatively in the condensed excitonic background [22].

In a typical excitonic semiconductor, the electrons in the conduction band are in an $s$-like state. For intermediate $n_{x}$ values, it is sufficient to consider the electron-heavy hole ( $h h$ ) coupling, with the $h h$ 's predominantly in $p$-like orbitals [23]. The SOCs for the electrons and the $h h$ 's are

$$
\begin{aligned}
& \mathcal{H}_{e}=i \alpha_{e} E_{z}\left(\sigma_{+} k_{-}-\sigma_{-} k_{+}\right), \\
& \mathcal{H}_{h}=i \beta_{h} E_{z}\left(\sigma_{+} k_{-}^{3}-\sigma_{-} k_{+}^{3}\right),
\end{aligned}
$$

where $\sigma_{ \pm}=\left(\sigma_{x} \pm i \sigma_{y}\right) / 2$ are the Pauli matrices and $k_{ \pm}=k_{x} \pm i k_{y}$ are the in-plane wave vectors. The SOCs $\alpha_{e}$ and $\beta_{h}$ can be inferred from many recent works $[15,23,24]$. However, the agreement on the suggested values is still lacking. The values for electrons vary from $\alpha_{e} \simeq$ $30.6 e \AA^{2}$ to $\alpha_{e} \simeq 300 e \AA^{2}$ in the range from $n_{x} \leq 1 \times$ $10^{11} \mathrm{~cm}^{-2}$ to $n_{x} \simeq 2.2 \times 10^{12} \mathrm{~cm}^{-2}$. For $h h$ 's the only results that the authors are aware of are by Winkler et al. [24] in which $\beta_{h}=7.5 \times 10^{6} e \AA^{4}$ for $n_{x} \leq 10^{11} \mathrm{~cm}^{-2}$. The calculated $\beta_{h}$ values are, however, found to be strongly dependent on the density [24]. The $E$ field strength at the interface is estimated by $E_{z}=e n_{x} /(2 \epsilon)$. Typical SOC energies for intermediate $n_{x}$ covering $10^{9}<n_{x}<$ $10^{11}\left(\mathrm{~cm}^{-2}\right)$ are perturbatively weaker than typical Coulomb energies at a given $n_{x}$ as shown in Table I. The electronic $r_{s}$ values vary in the range $1 \leq r_{s}<500$. The crossover regime $[2,3,13]$ characterized by $r_{s} \simeq 2-5$ from the strongly interacting BEC to the weakly interacting BCS type condensation can therefore be probed by the strength of the SOC.

For $n_{x}<10^{11} \mathrm{~cm}^{-2}$, only the lowest $h h$ states are occupied in the valence band [23]. For $n_{x} \leq 10^{9} \mathrm{~cm}^{-2}$ the spin dependent splitting is difficult to observe [25]. We therefore consider here the range $10^{9} \leq n_{x} \leq 10^{11}\left(\mathrm{~cm}^{-2}\right)$.

In the absence of SOC the condensed state is formulated by the $e$ - $h h$ quasiparticle eigenstates $\hat{\alpha}_{\vec{k}, \sigma}=$ $\cos \left(\theta_{\vec{k}} / 2\right) \hat{c}_{\vec{k}, \sigma}-\sin \left(\theta_{\vec{k}} / 2\right) \hat{d}_{-\vec{k}, \sigma}^{\dagger} ; \quad \hat{\beta}_{\vec{k}, \sigma}=\sin \left(\theta_{\vec{k}} / 2\right) \hat{c}_{\vec{k}, \sigma}+$ $\cos \left(\theta_{\vec{k}} / 2\right) \hat{d}_{-\vec{k}, \sigma}^{\dagger}$, where $\vec{k}=\left(k_{x}, k_{y}\right), \sigma=\uparrow, \downarrow$, and $\hat{c}_{\vec{k}, \sigma}$ and $\hat{d}_{\vec{k}, \sigma}$ are the annihilation operators for the electron and the $h h$. The cosine and sine coherence factors have been found [13] for the geometry of Fig. 1 using the HF

TABLE I. Interface $E$ fields and SOC energies for typical densities.

\begin{tabular}{lcccc}
\hline \hline$n_{x}\left(\mathrm{~cm}^{-2}\right)$ & $E_{z}(\mathrm{kV} / \mathrm{cm})$ & $\alpha_{e} k_{F} E_{z}(\mathrm{meV})$ & $\beta_{h} k_{F}^{3} E_{z}(\mathrm{meV})$ & $\beta_{h} k_{F}^{2} / \alpha_{e}$ \\
\hline $10^{9}$ & 1.45 & $5 \times 10^{-4}$ & $1.5 \times 10^{-4}$ & 0.3 \\
$10^{10}$ & 14.5 & $1.5 \times 10^{-2}$ & $4.9 \times 10^{-2}$ & 3.3 \\
$10^{11 \mathrm{a}}$ & 145 & 0.8 & 15 & 18.75 \\
$10^{12 \mathrm{~b}}$ & $1.45 \times 10^{3}$ & 154 & $4.8 \times 10^{3}$ & 31 \\
\hline \hline
\end{tabular}

${ }^{\mathrm{a}}$ Reference [23].

${ }^{\mathrm{b}}$ Reference [15]. mean-field of the real excitonic order parameter (EOP)

$$
\Delta_{0}^{\left(\sigma \sigma^{\prime}\right)}(\vec{k})=\sum_{\vec{k}^{\prime}} V_{\vec{k}-\vec{k}^{\prime}}^{e h}\left\langle\hat{c}_{\vec{k}^{\prime}, \sigma}^{\dagger} \hat{d}_{-\vec{k}^{\prime}, \sigma^{\prime}}^{\dagger}\right\rangle .
$$

Because of the rotational invariance of the momentum and the spin spaces, the ground state is isotropic and spin independent [26]. At low $n_{x}$, the EOP is large near $k=0$ and the condensation is BEC type [13]. For increasing $n_{x}$ the peak position shifts to a finite value near $k_{F}$ where the BCS type pairing is dominant [14].

Including the SOC, the time reversal symmetry remains but the spin degeneracy is lifted. The full Hamiltonian, in the basis $\left(\hat{\alpha}_{\vec{k}, \uparrow} \hat{\beta}_{-\vec{k}, \uparrow}^{\dagger} \hat{\alpha}_{\vec{k}, \downarrow} \hat{\beta}_{-\vec{k}, \downarrow}^{\dagger}\right)$, is then

$$
\mathcal{H}=\left(\begin{array}{cccc}
-E_{\vec{k}} & 0 & i A+\Delta_{1} & i C+\Delta_{2} \\
0 & E_{\vec{k}} & -i C+\Delta_{3}^{*} & i B-\Delta_{1} \\
-i A^{*}+\Delta_{1}^{*} & i C^{*}+\Delta_{3} & -E_{\vec{k}} & 0 \\
-i C^{*}+\Delta_{2}^{*} & -i B^{*}-\Delta_{1}^{*} & 0 & E_{\vec{k}}
\end{array}\right) \text {, }
$$

where the diagonal terms correspond to the lower $\left(\hat{\alpha}_{\vec{k}, \sigma}\right)$ and the upper $\left(\hat{\beta}_{\vec{k}, \sigma}\right)$ excitonic bands determined by [13]

$$
\begin{aligned}
E_{\vec{k}} & =\sqrt{\zeta_{\vec{k}}^{2}+\Delta_{0}^{2}(\vec{k})} \\
\zeta_{\vec{k}} & =E_{g} / 2+\epsilon_{\vec{k}}-\mu_{x}+\sum_{\vec{k}^{\prime}} V_{\vec{k}-\vec{k}^{\prime}} \zeta_{\vec{k}^{\prime}} / E_{\vec{k}^{\prime}} \\
& =E_{\vec{k}} \cos \theta_{\vec{k}} \Delta_{0}(\vec{k})=\frac{1}{2} \sum_{\vec{k}^{\prime}} V_{\vec{k}-\vec{k}^{\prime}}^{e h} \Delta_{0}\left(\vec{k}^{\prime}\right) / E_{\vec{k}^{\prime}}=E_{\vec{k}} \sin \theta_{\vec{k}} \\
n_{x} & =\frac{1}{2} \sum_{\vec{k}^{\prime}}\left(1-\zeta_{\vec{k}^{\prime}} / E_{\vec{k}^{\prime}}\right) .
\end{aligned}
$$

Here $\mu_{x}$ is the exciton chemical potential. In (3) $A$ and $B$ are the intraband excitonic SOCs for the lower and the upper branches and $C$ is the interband SOC. The higher excitonic band can be neglected here since the $\hat{\beta}$ states contribute to the $\hat{\alpha}$ state intraband transition energies on the order of $|C|^{2} / \Delta_{0}^{2}$ for low momenta, and $|C|^{2} / \zeta_{\vec{k}}^{2}$ for high momenta, which are both negligible. Eliminating the $\hat{\beta}$-like states, (3) can be reduced to a $2 \times 2$ matrix for the lower band where only $A$ and $\Delta_{1}$ are relevant which are

$$
A(\vec{k})=i E_{z}\left[\alpha_{e} \cos ^{2}\left(\theta_{\vec{k}} / 2\right) k_{-}+\beta_{h} \sin ^{2}\left(\theta_{\vec{k}} / 2\right) k_{-}^{3}\right]
$$

$$
\Delta_{1}(\vec{k})=\cos \left(\theta_{\vec{k}} / 2\right) \sin \left(\theta_{\vec{k}} / 2\right)\left[\Delta_{\uparrow \downarrow}^{*}(\vec{k})+\Delta_{\downarrow \uparrow}(\vec{k})\right],
$$

where $\Delta_{\downarrow \uparrow}(\vec{k})=\sum_{\vec{k}^{\prime}} V_{\vec{k}-\vec{k}^{\prime}}^{e h}\left\langle\hat{c}_{\vec{k}^{\prime}, \downarrow}^{\dagger} \hat{d}_{-\vec{k}^{\prime}, \uparrow}^{\dagger}\right\rangle$ is the complex excitonic spin-orbit order parameter (ESOOP). For this lower branch, the SOC-split eigenenergies are

$$
\lambda_{\vec{k}}^{ \pm}=-E_{\vec{k}} \pm \Delta E_{\vec{k}}, \quad \Delta E_{\vec{k}}=\left|i A(\vec{k})+\Delta_{1}(\vec{k})\right|,
$$

where the eigenstates indexed by \pm are 


$$
\hat{\eta}_{\vec{k}, \pm} \rightarrow \frac{1}{\sqrt{2}}\left(\begin{array}{c}
1 \\
\pm e^{i \Lambda_{\vec{k}}}
\end{array}\right)
$$

in the $\left(\hat{\alpha}_{\vec{k},}, \hat{\alpha}_{\vec{k}, \downarrow}\right)$ basis, and the relative phase is

$$
e^{i \Lambda_{\vec{k}}}=\left[i A(\vec{k})+\Delta_{1}(\vec{k})\right] /\left[\left|i A(\vec{k})+\Delta_{1}(\vec{k})\right|\right] .
$$

The complex ESOOP is then calculated by Eq. (6) as

$$
\Delta_{1}(\vec{k})=\frac{1}{4} \frac{\Delta_{0}(\vec{k})}{E_{\vec{k}}} \sum_{\vec{k}^{\prime}} e^{i \Lambda_{\vec{k}^{\prime}}} V_{\vec{k}-\vec{k}^{\prime}}^{e h} \frac{\Delta_{0}\left(\vec{k}^{\prime}\right)}{E_{\vec{k}^{\prime}}} .
$$

Equations (5), (6), (9), and (10) form a self-consistent set describing the effect of the SOC and they depend on the solutions of (4). There is a $C_{\infty v}$ symmetry respected by the electronic part of the Hamiltonian [11] which arises due to continuous rotations in $\vec{k}$ space and the double covering of the spin- $1 / 2$ representation. On the other hand, the SOC for the $h h s$ has a cubic momentum dependence in contrast to the linear one in the electronic SOC. Additionally, the spin space of the $h h s$ (i.e., $S=3 / 2, S_{z}= \pm 3 / 2$ ) is incomplete in the spin-3/2 representation. Therefore, the hole SOC breaks the electronic $C_{\infty v}$ and this has observable consequences.

The phase of $\Delta_{1}$ is plotted in Fig. 2. We observe that at relatively high $n_{x}\left(\simeq 1.7 \times 10^{11} \mathrm{~cm}^{-2}\right.$ here $)$, the ESOOP phase is relatively coherent for weak electric fields, i.e., Fig. 2(a), despite the strong phase variations in the SOC. We attribute this to the dominant contribution in (10) near the Fermi level $[13,14]$, where $\Delta_{0}\left(k_{F}\right) / E_{k_{F}} \simeq 1$. There, the SOC dictates the phase profile due to a high density of states (DOS). Thus, the increase in the $E$ field has a
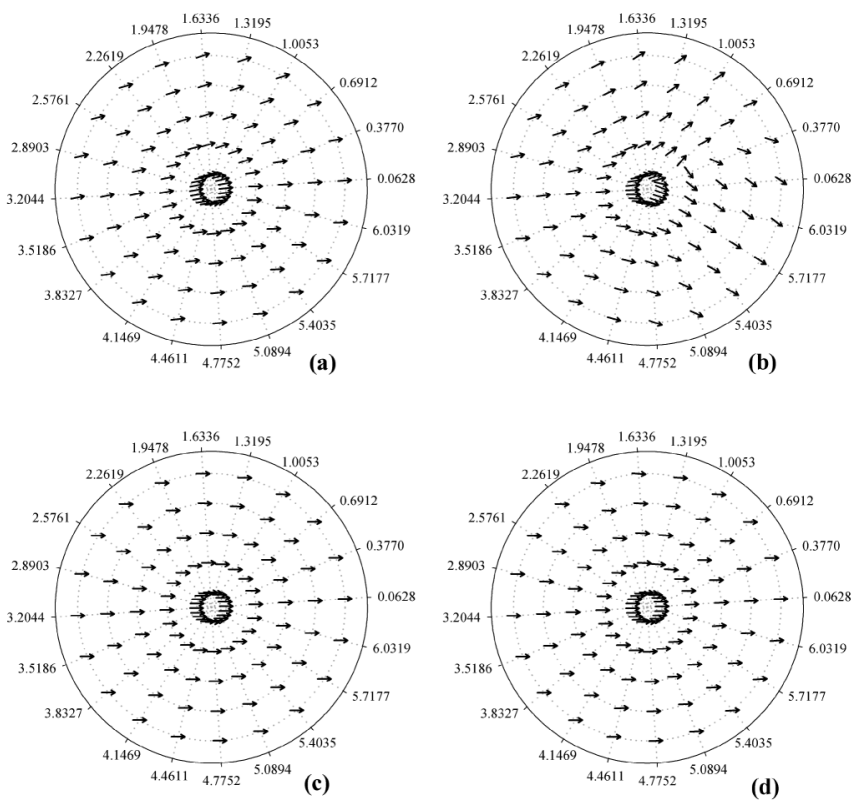

FIG. 2. Phase of $\Delta_{1}$ is shown in $\vec{k}$ for $n_{x}=1.7 \times 10^{11} \mathrm{~cm}^{-2}$ with $E_{z}=15 \mathrm{kV} / \mathrm{cm}$ (a) and $E_{z}=150 \mathrm{kV} / \mathrm{cm}$ (b), $n_{x}=$ $10^{10} \mathrm{~cm}^{-2}$ with $E_{z}=15 \mathrm{kV} / \mathrm{cm}$ (c), and $E_{z}=150 \mathrm{kV} / \mathrm{cm}$ (d). The radial range is $0 \leq k \leq 3$ in units of $a_{e}^{*} \simeq 100 \AA$. significant effect as observed in Fig. 2(b). At lower $n_{x}$ $\left(\simeq 10^{10} \mathrm{~cm}^{-2}\right.$ here), there is a weak overlap between the condensed pairs and the dominant contribution to (10) is near $k=0$, where $\Delta_{0}(\vec{k}=0) / E_{\vec{k}=0} \simeq 1$. The DOS has a minimum there and a small number of states cannot accommodate the anisotropy in the weak SOC. Thus the phase rigidity is imposed by the dominant Coulomb interaction, as in Fig. 2(c). There, the phase is less sensitive to the $E$-field strength of the already weak SOC [Fig. 2(d)].

The corresponding solutions for $\left|\lambda_{\vec{k}}^{(-)}\right|$in Fig. 3 demonstrate that the rotational symmetry of the ground state is broken by the anisotropic phase of the SOC. This should be compared with the isotropic results previously calculated $[13,14]$ without the SOC. The difference is made by $\Delta E_{\vec{k}}$ in (7) and it is an interference effect as shown below. From Fig. 2 we know that for high $n_{x}$, both $\Delta_{1}$ and $A$ are anisotropic and phase incoherent. Hence an interference is observed in $\left|i A+\Delta_{1}\right|$ between these two terms [Fig. 3(a) and 3(b)]. In the opposite limit of low $n_{x}$ as shown in Fig. 3(c) and 3(d) the phase of $\Delta_{1}$ is uniform but the SOC contribution is much weaker. Hence the energy profile is nearly isotropic.

Other features of Fig. 3 are similar to the case without the SOC. At higher $n_{x}$ the spin-independent EOP has a maximum $[13,14]$ and $\left|\lambda_{\vec{k}}\right|$ develops a minimum in the vicinity of the $k_{\min } \simeq 1$ ring created by the pure excitonic term in (7), i.e., a BCS type pairing. In the presence of the SOC, this ring shaped minimum is deformed as shown in Fig. 3(a) and 3(b). For lower $n_{x}$, as shown in Fig. 3(c) and $3(\mathrm{~d})$, the spin-independent EOP is maximum and $\left|\lambda_{\vec{k}}\right|$ is minimum at $k_{\min }=0$, i.e., a BEC type pairing. With the
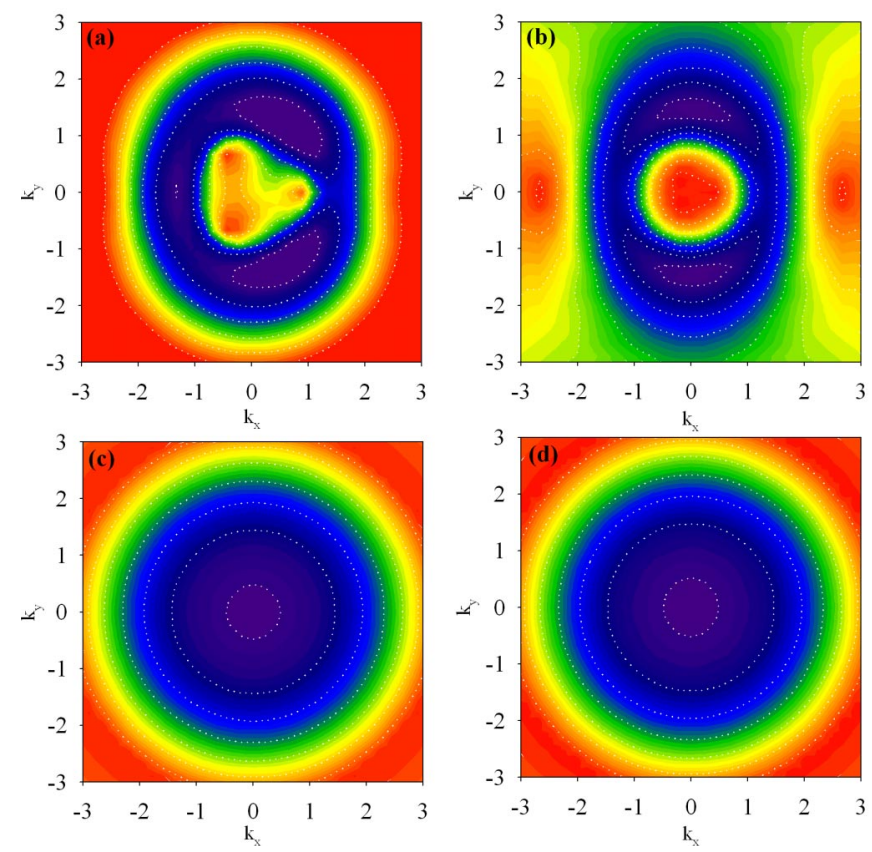

FIG. 3 (color online). Lower excitonic band $\left(\lambda_{\vec{k}}^{-}\right)$is shown here for the same $E_{z}$ and $n_{x}$ values and in the same order as in Fig. 2 above. The darker colors mean lower values. 
SOC, the additional splitting given by $\left|i A+\Delta_{1}\right|$ is also isotropic and does not deform the isotropic contribution of the spin-independent part.

From the experimental point of view, the off-diagonal components of the static spin susceptibility $\chi_{i j}(\star)$, where $\star=\left(\vec{q} \rightarrow 0, i \omega_{n}=0\right)$, reveal the complex ESOOP and the breaking of the $C_{\infty v}$ symmetry [11]. The $\chi_{i j}(\star)$ is

$$
\chi_{i j}(\star)=\mu_{B}^{2} \lim _{\vec{q} \rightarrow 0} \int_{0}^{1 / T} d \tau\left\langle T_{\tau} \hat{m}_{i}(\vec{q}, \tau) \hat{m}_{j}(-\vec{q}, 0)\right\rangle,
$$

where $\mu_{B}$ is the effective Bohr magneton, $\tau$ is the Matsubara time, $T_{\tau}$ is the time ordering operator, $T$ is the temperature, $\hat{m}_{i}(\vec{q}, \tau)=\sum_{\vec{k}, \mu \nu} \hat{\alpha}_{\vec{k}+\vec{q}, \mu}^{\dagger}(\tau)\left[\sigma_{i}\right]^{\mu \nu} \hat{\alpha}_{\vec{k}, \nu}(\tau)$ is the magnetization operator in the lower excitonic branch, and $\nu_{F}$ is the DOS at the Fermi level. We focus on the offdiagonal terms in the limit $T \rightarrow 0$, as those have the strongest signature of the $C_{\infty v}$ breaking for which we find,

$$
\begin{gathered}
\frac{\chi_{z x}(\star)+i \chi_{z y}(\star)}{\chi_{P}} \simeq-\left.\frac{1}{4} \frac{\partial}{\partial E_{\vec{k}}}\left\langle i A+\Delta_{1}\right\rangle_{a}\right|_{E_{\vec{k}}=E_{F}} \\
\left.\frac{\chi_{x y}(\star)}{\chi_{P}} \simeq \frac{1}{6} \frac{\partial^{2}}{\partial E_{\vec{k}}^{2}} \widetilde{I} m\left\{\left\langle\left(i A+\Delta_{1}\right)^{2}\right\rangle_{a}\right\}\right|_{E_{\vec{k}}=E_{F}},
\end{gathered}
$$

where $\langle\ldots\rangle_{a}$ is the angular average and $\chi_{P}$ is the Pauli paramagnetic susceptibility. If the Fermi contour is isotropic, (12) and (13) both vanish. This occurs at low $n_{x}$ [i.e., (c) and (d) in Figs. 2 and 3], where the phase of $\Delta_{1}$ is coherent and $\left|\Delta_{1}\right|$ is isotropic. On the other hand, at higher $n_{x}$ the Fermi contour is anisotropic [i.e., (a) and (b) in Figs. 2 and 3] and the phase of $\Delta_{1}$ varies. Therefore, the effect in (12) and (13) may be visible within the BCS limit at relatively high $n_{x}$. Considering that the magnitude of $\Delta_{1}$ is set by the $e-h$ Coulomb interaction, we approximately have $\left(\chi_{z x}+i \chi_{z y}\right) / \chi_{P} \simeq V_{\vec{q}_{F}}^{e h} / E_{F} \leq 0.1$ and $\chi_{x y} / \chi_{P} \simeq$ $\left(V_{\vec{q}_{F}}^{e h} / E_{F}\right)^{2} \leq 0.01$.

In conclusion, in the presence of excitonic background, the interference between the electron and the hole SOCs renders the $e$ - $h$ pairing unconventional by breaking the rotational symmetry of the ground state. The resulting complex order parameter is affected by the exciton density. As the density is increased, the magnitude smoothly changes from an isotropic BEC type to an anisotropic BCS type. On the other hand, its phase is globally coherent at low densities, and it gradually becomes nonuniform at increased densities. The predicted strength is small but observable in the off-diagonal static spin susceptibility, suggesting a new direction in the experimental observation of the excitonic condensate.

We thank J. Schliemann for discussions. This research is supported by TÜBITAK Grants No. 105T110; No. B.02.1.TBT.0.06.03.00/1120/2985. The susceptibility part was carried out at the Marmaris Institute of Theoretical and Applied Physics (ITAP).
[1] S. A. Moskalenko, Fizicheskie metody issledovaniia tverdogo tela / Uralskii Politekhnicheskii Institut im SM Kirova 4, 276 (1962); J. M. Blatt, K. W. Böer, and W. Brandt, Phys. Rev. 126, 1691 (1962); L. V. Keldysh and Yu. V. Kopaev, Sov. Phys. Solid State 6, 2219 (1965); L. V. Keldysh and A. N. Kozlov, JETP 27, 521 (1968); R. R. Guseinov and L. V. Keldysh, JETP 36, 1193 (1973).

[2] C. Comte and P. Noziéres, J. Phys. (France) 43, 1069 (1982); P. Noziéres and C. Comte, J. Phys. (France) 43, 1083 (1982).

[3] R. Cote and A. Grifin, Phys. Rev. B 37, 4539 (1988).

[4] Bose-Einstein Condensation of Excitons and Biexcitons, edited by S. A. Moskalenko and D. W. Snoke (Cambridge University Press, Cambridge, England, 2000).

[5] D. W. Snoke and J. P. Wolfe, Phys. Rev. B 39, 4030 (1989); D. W. Snoke, J.P. Wolfe, and A. Mysyrowicz, Phys. Rev. B 41, 11171 (1990).

[6] K.E. O’Hara, L. O. Suilleabhain, and J. P. Wolfe, Phys. Rev. B 60, 10565 (1999).

[7] L. V. Butov et al., Phys. Rev. Lett. 86, 5608 (2001); J. P. Cheng et al., Phys. Rev. Lett. 74, 450 (1995).

[8] T. Fukuzawa et al., Surf. Sci. 228, 482 (1990); T. Fukuzawa, E. E. Mendez, and J. M. Hong, Phys. Rev. Lett. 64, 3066 (1990); L. V. Butov et al., Phys. Rev. Lett. 73, 304 (1994); Phys. Rev. B 52, 12153 (1995); A. Zrenner, L. V. Butov, and M. Hagn, Semicond. Sci. Technol. 9, 1983 (1994).

[9] A. V. Gorbunov and V. B. Timofeev, Phys. Stat. Sol. 2, 871 (2005); D. W. Snoke et al., Solid State Commun. 134, 37 (2005); J.P. Eisenstein and A.H. MacDonald, Nature (London) 432, 691 (2004).

[10] E. I. Rashba, Sov. Phys. Solid State 2, 1109 (1960); Yu. A. Bychkov and E. I. Rashba, JETP Lett. 39, 78 (1984).

[11] Lev P. Gorkov and Emmanuel I. Rashba, Phys. Rev. Lett. 87, 037004 (2001).

[12] R. Balian and N. R. Werthamer, Phys. Rev. 131, 1553 (1963); Manfred Sigrist and Kazuo Ueda, Rev. Mod. Phys. 63, 239 (1991).

[13] X. Zhu et al., Phys. Rev. Lett. 74, 1633 (1995).

[14] P. B. Littlewood et al., J. Phys. Condens. Matter 16, S3597 (2004); P. B. Littlewood and Xuejun Zhu, Phys. Scr. T68, 56 (1996).

[15] G. Engels et al., Phys. Rev. B 55, R1958 (1997); T. Matsuyama et al., Phys. Rev. B 61, 15588 (2000).

[16] E. A. de Andrada e Silva, G. C. La Rocca, and F. Bassani, Phys. Rev. B 55, 16293 (1997).

[17] J. Nitta et al., Phys. Rev. Lett. 78, 1335 (1997).

[18] J. P. Lu et al., Phys. Rev. Lett. 81, 1282 (1998).

[19] Dirk Grundler, Phys. Rev. Lett. 84, 6074 (2000).

[20] S. J. Papadakis et al., Science 283, 2056 (1999).

[21] B. Datta and S. Das, Appl. Phys. Lett. 56, 665 (1990).

[22] This approach is opposite to the case in Ref. [11] where SOC is stronger than the typical condensation energy.

[23] R. Winkler, Phys. Rev. B 62, 4245 (2000).

[24] R. Winkler, H. Noh, E. Tutuc, and M. Shayegan, Phys. Rev. B 65, 155303 (2002).

[25] R. Winkler, H. Noh, E. Tutuc, and M. Shayegan, Phys. Rev. B 65, 155303 (2002).

[26] B. I. Halperin and T. M. Rice, Solid State Physics, edited by F. Seitz, D. Turnbull, and H. Ehrenfest (Academic, New York, 1956), Vol 21, p. 115. 